\title{
LA PROMOCIÓN DE VALORES A TRAVÉS DE UN PROGRAMA DE ACTIVIDAD FÍSICA Y PREDEPORTES EN PERSONAS CON DISCAPACIDAD INTELECTUAL LEVE
}

\section{Promoting values through a physical and pre-sport activity programme in people with intellectual disabilities}

Elisa Isabel SÁNCHEZ-ROMERO

Universidad Católica de Murcia. Departamento de Ciencias Sociales, Jurídicas y de la Empresa. Campus de los Jerónimos, 135. Guadalupe 30107. Murcia

eisanchez@ucam.edu

M. Pilar Vílchez CONESA

Universidad Católica de Murcia. Departamento de Ciencias Sociales, Jurídicas y de la Empresa

Cristina De Francisco Palacios

Universidad Católica de Murcia. Departamento de Ciencias Sociales, Jurídicas y de la Empresa

Recepción: 20 de septiembre de 2016

Fecha de aceptación definitiva: 21 de julio de 2017

RESUMEN: En este trabajo se presenta un programa diseñado para la promoción de valores en personas con discapacidad intelectual leve, a través de sesiones de actividad física y predeporte, en un contexto educativo dirigido a su inserción sociolaboral. La intervención se aplicó a una muestra de 12 participantes. El instrumento utilizado fue una adaptación de la versión española del Sport Value Questionnaire (SVQ-E), además de un registro observacional para la recogida de datos en los debates posteriores a los dilemas. Las opiniones del alumnado mejoraron respecto a cada valor después del programa. Además, los resultados indican que los sujetos consideraban más importantes los valores trabajados en el programa que los no trabajados.

Palabras Clave: valores; dilemas; actividad física; discapacidad intelectual. 
ABSTRACT: This study presents an intervention program in order to promote values in people with mild intellectual disabilities. The program is structured in several sessions of physical activity and adapted physical/sporting activities in an educational context directed to socio-labor insertion of collective with mild intellectual disabilities. The intervention was applied to a sample of 12 participants. The Spanish version of the Sport Value Questionnaire (SVQ-E) was the measurement instrument as well as recording observations for collecting data in the post-dilemma debates an observational record for collecting data in post-dilemma debates. Judgment of the students improved with respect to each value after the program. In addition, results indicate that subjects considered more important the values of the program than the ones not worked.

KEYwORDs: values; dilemmas; physical activity; intellectual disability.

\section{Introducción}

L

OS VALORES PUEDEN ENTENDERSE como aquellos principios y convicciones, estables en las distintas situaciones, que guían el comportamiento de las personas ( La sociedad actual está asistiendo a una preocupante crisis de valores, por lo que la formación en valores se perfila como algo necesario ante la ausencia de consenso en la transmisión de los mismos (Pombo y Fantova, 2011). Educar en valores proporciona herramientas para la paz y el desarrollo en las personas, formando a ciudadanos responsables y justos (Peter, 2014), haciéndose necesario que las organizaciones de aprendizaje posean un sistema de valores para apoyar la formación que se da en las mismas (Schalock y Verdugo, 2007).

En el ámbito educativo, el deporte se erige como un medio idóneo para la educación en valores (Borrás, Palou, Ponseti, Vidal y García-Mas, 2009; Martínez et al., 2014), encontrando una relación positiva entre la moralidad trabajada en educación física y la de la vida en sociedad (Parisi, Mouratidou, Koidou, Tsorbatzoudis y Karamavrou, 2015). En el contexto universitario, Ionescu (2012) afirma que la educación física es un factor clave en la formación de futuros ciudadanos en términos de desarrollo de su personalidad. Estudios similares han obtenido mejoras significativas en los valores que se trabajaron a través de la actividad físico-deportiva en adolescentes (Borrás et al., 2009).

En el escenario educativo formal son múltiples las investigaciones que se han llevado a cabo sobre el proceso de adquisición de valores a través del deporte, en Educación Primaria (Hernández-Mendo y Planchuelo, 2014), en Educación Secundaria Obligatoria (Barba, Barba y Muriarte, 2003; Martínez et al., 2014) y en Educación Superior (Barba y Matías, 2005; Benítez, 2009). Sin embargo, los alumnos con discapacidad intelectual no han sido un colectivo en el que se haya evaluado la adquisición de valores (Langdon, Clare y Murphy, 2010) ya que, tras finalizar la enseñanza básica, los programas formativos para este colectivo son escasos (Fullana, Pallisera, Martín, Ferrer y Puyaltó, 2015).

Por todo ello, el escenario de la educación superior se convierte en un lugar adecuado para desarrollar programas dirigidos a personas adultas con discapacidad intelectual 
(Cerrillo, Izuzquina y Egido, 2013). Con el objetivo de la inserción laboral, estos programas aumentan la confianza y deseo de autosuperación en los adultos con discapacidad intelectual, fomentando habilidades sociales necesarias para su desenvolvimiento en diferentes ámbitos de la vida, ayudando a su integración en el contexto social (Izuzquiza, 2012; Molina, Vargas y San Martín, 2014).

\section{Objetivos}

1. Promover diferentes valores a través de un programa de actividad física y predeportes en personas con discapacidad intelectual leve.

2. Comparar los resultados obtenidos en los valores incluidos en el programa de actividad física y predeportes con otros valores no trabajados en el programa.

\section{Método}

\subsection{Sujetos}

La muestra estuvo formada por los 12 estudiantes de la asignatura "Valores, tiempo libre y deporte" de un programa destinado a la inclusión sociolaboral de personas con discapacidad intelectual leve. Los participantes tenían una media de edad de 24,67 $\pm 5,22$. Todos ellos presentaban una discapacidad reconocida del $33 \%$ o más (concretamente entre 33 y 75 grados) y una conducta social ajustada. Un 8,3\% de los participantes finalizaron un Ciclo Formativo de Grado Medio, y un 91,7\% finalizaron Programas de Cualificación Profesional Inicial (PCPI) o estudios de Enseñanza Secundaria Obligatoria (ESO), a través de programas específicos de atención a la diversidad. Ningún estudiante padecía enfermedades físicas relevantes para la práctica de actividad físico-deportiva, por lo que no hubo que adaptar las sesiones planificadas.

\subsection{Descripción de la intervención}

Los valores que se trabajaron fueron compromiso, compañerismo, respeto, igualdad, honestidad/fair play y responsabilidad. A continuación, se establece la definición de cada uno de los valores, tal y como se entendieron en el programa:

- Compromiso. El valor compromiso se entiende como la creencia firme en una meta objeto del compromiso, por lo que una persona comprometida cumplirá voluntariamente las normas y expectativas, esforzándose en ocasiones por el bien del objeto del compromiso (Firestone y Pennell, 1993).

- Compañerismo. El valor compañerismo nace de la proximidad física continuada con alguien, ya sea en el contexto escolar, el lugar de trabajo o la pertenencia 
LA PROMOCIÓN DE VALORES A TRAVÉS DE UN PROGRAMA DE ACTIVIDAD FÍSICA Y PREDEPORTES EN PERSONAS CON DISCAPACIDAD INTELECTUAL LEVE

ELISA ISABEL SÁNCHEZ-ROMERO, M. ${ }^{a}$ PILAR VÍLCHEZ CONESA Y CRISTINA DE FRANCISCO PALACIOS

a un grupo social, y deriva en el sentimiento de vinculación y de aprecio hacia el otro (García-Hoz, 1993).

- Respeto. El valor respeto se concibe como la disposición social que deja ser, a cada persona, lo que quiere ser. En este sentido, respetar es conceder autonomía, aceptando al otro, aunque no lo entendamos, reconociendo el valor que tiene (Naval, 2005).

- Igualdad. Este valor se manifiesta cuando las personas experimentan que todos tienen el mismo valor como persona y los mismos derechos que los demás (Hall, 1995).

- Honestidad/fair play. Las personas, cuando asumen este valor, basan su conducta en la noción de justicia, por lo que nunca pretenderán tener ventajas, de manera injusta, ni de forma intencional ni fortuita (Cruz, Boixadós, Valiente y Torregrosa, 2001).

- Responsabilidad. Según Ortega y Mínguez (2003), es más que dar cuenta de nuestros comportamientos, ya que comprende el ámbito de la atención hacia los demás, respondiendo hacia el otro. Además, el valor responsabilidad, en este programa, se entiende como lo opuesto a la indiferencia hacia sí mismo y hacia los demás.

Cada valor se trabajó en dos sesiones de actividad física en formato de predeporte, a excepción del primer valor, el compromiso, que se desarrolló en tres, utilizando la primera sesión como toma de contacto y análisis de las características físicas e intelectuales de los alumnos.

Todas las sesiones se componían de tres partes, calentamiento, parte principal y vuelta a la calma. En la parte principal de las primeras sesiones se trabajaron aspectos técnicos de cada predeporte, mientras que, en la parte principal de las segundas sesiones, se proponían situaciones jugadas que trabajaran cada uno de los valores. Todos los aspectos importantes que se producían en esta situación jugada eran tratados posteriormente. Se iniciaba el debate con una historia similar en una situación deportiva, tras la que los alumnos comentaban sus opiniones y se les ponían ejemplos acerca de situaciones diseñadas por el profesorado que habían vivido ellos en la sesión.

El primer valor, el compromiso, se trabajó a través del voleibol. Se trabajaron situaciones cooperativas en seis equipos de dos componentes, teniendo que hacer más toques seguidos que otros compañeros en la misma situación de dos con dos. El compromiso se mostraba cuando los alumnos eran conscientes de que el equipo necesitaba a cada componente para conseguir desarrollar la tarea cooperativa. El dilema que inició el debate para el trabajo del compromiso, tras la sesión, fue el siguiente:

Miguel tiene dificultades para correr, pero se ha esforzado mucho durante la clase de hoy. La profesora divide al grupo en dos equipos para jugar un partido y selecciona a Rosa y a Juan para ejercer de entrenadores. Miguel está en el equipo de Rosa. Esta le comenta que no se preocupe, que durante el partido dará oportunidades a todos los jugadores del equipo, porque participar es más importante que el resultado. Pasan los minutos y Miguel sigue en el banquillo, pero el otro equipo está ganando. Si saca a Miguel puede perder el partido. ¿Debe Rosa sacar a Miguel sabiendo que su equipo perderá el partido?

(C) Ediciones Universidad de Salamanca / CC BY-NC-ND

Siglo Cero, vol. 48 (3), n. ${ }^{\circ}$ 263, 2017, julio-septiembre, pp. 73-86 
El segundo valor, el compañerismo, fue trabajado a través del bádminton. La situación jugada para el trabajo de este valor consistía en hacer tres equipos de cuatro jugadores, donde jugaban dos equipos mientras uno de ellos observaba el partido desde fuera y arbitraba. Dadas las dificultades de algunos alumnos ante el saque, se observaba el comportamiento de los demás para, posteriormente, conectarlo con el valor del compañerismo a comentar tras la sesión. El dilema que inició el debate para el trabajo del compañerismo fue el siguiente:

En la última clase se ha enseñado bádminton (como nosotros) a los alumnos y la profesora decide que hoy harán un examen práctico en situación de competición. Enrique es un buen jugador, de hecho, la profesora en la última clase le ha felicitado en múltiples ocasiones. En el reparto de contrincantes para el examen, a Enrique lo han emparejado con María, compañera a la que no se le da muy bien el bádminton. Comienza el examen y Enrique gana continuamente puntos, no dejando jugar a María. Enrique decide perder un par de saques para que María pueda aprobar el examen. La profesora se da cuenta y le dice a Enrique que no puede dejar ganar a María, engañar no está bien y si sigue actuando así, suspenderá a Enrique. Enrique no sabe qué hacer. ¿Debe Enrique hacer caso a su profesora y no dejar que María pueda demostrar su juego?

El tercer valor, el respeto, se trabajó con balonmano. La situación jugada consistió en formar dos equipos de cuatro componentes más el portero, siendo árbitro un alumno de cada equipo (se iban alternando). Para que el gol fuese válido, todos los compañeros del equipo debían haber recibido al menos un pase. El dilema que inició el debate para el trabajo del respeto fue el siguiente:

Jugando al balonmano, Eva ha recibido un codazo intencionado de Pedro que quería evitar que tirase a puerta. El árbitro no ha visto el codazo por lo que Eva se dirige a él pidiendo que pite falta. El árbitro le contesta que él no lo ha visto, Eva sigue insistiendo, pese a saber que a ese árbitro en particular no le gustan las protestas y suele sacar tarjeta frente a ellas. Eva puede resultar expulsada de la pista perjudicando al equipo. Insiste tanto que llega a decirle al árbitro si es miope, si no tiene ojos en la cara, etc., ¿¿debe Eva seguir insistiendo?

El cuarto valor, la igualdad, se trabajó con fútbol sala, dado que es un deporte donde suele haber más diferencia de habilidad entre géneros debido a la práctica (Otero-Pazos, Navarro-Patón y Abelairas-Gómez, 2014). Se formaron dos equipos de cinco componentes más el portero, donde uno de cada equipo tenía que hacer de entrenador. El propio equipo decidía quién hacía de entrenador, con la única condición de que no podían ser las chicas, estas siempre tenían que ser jugadoras. Además, para que el gol fuese válido, todos los jugadores tenían que haber tocado el balón antes. El dilema que inició el debate para el trabajo de la igualdad fue el que se presenta a continuación: “A Ana le gusta mucho el fútbol, pero no se le da muy bien. Aun así, quiere jugar y aprender, así que se apunta a un club que está jugando una liga regional mixta (chicos y chicas). Van ganando partidos y se encuentran en la final, el partido más importante. ¿Debe el entrenador sacar a Ana para que juegue el partido o no porque juega peor que sus compañeros y es la final regional?”. 
LA PROMOCIÓN DE VALORES A TRAVÉS DE UN PROGRAMA DE ACTIVIDAD FÍSICA Y PREDEPORTES EN PERSONAS CON DISCAPACIDAD INTELECTUAL LEVE

ELISA ISABEL SÁNCHEZ-ROMERO, M. ${ }^{a}$ PILAR VÍLCHEZ CONESA Y CRISTINA DE FRANCISCO PALACIOS

El quinto valor, la honestidad/fair play, se trabajó a través de "fut-tenis", en un juego de dos contra dos. Se utilizaban las reglas del tenis, pero golpeando el balón con el pie y, en algunos casos (como el saque), con la mano. El jugador que tenía más cerca la línea tenía que decir si había sido dentro o fuera en acuerdo con el resto de jugadores, aceptando a su vez todas las normas propuestas. El dilema que inició el debate para el trabajo de la honestidad/fair play fue el que sigue:

Alfonso odiaba perder a lo que fuera. Se sentía tan bien cuando ganaba, que no quería renunciar a aquella sensación por nada del mundo; además, cuando perdía, le parecía lo peor que a uno le puede ocurrir. Por eso no jugaba a nada que no se le diera muy bien. Cuando conoció a Alberto, un chico nuevo excelente jugador, no tardaron en enfrentarse. Alfonso se preparó concentrado y serio, dispuesto a ganar, pero Alberto no parecía tomárselo en serio, andaba todo el rato sonriente y hacía chistes, sobre todo. Pero era realmente un fenómeno, marcaba goles una y otra vez, y no paraba de reír. Estaba tan poco atento, que Alfonso pudo hacerle trampas con el marcador, incluso como para ganar el partido. Pero Alfonso no se sentía bien con esa victoria, ¿por qué?

El sexto y último valor a trabajar en el programa de intervención fue la responsabilidad. Se trabajó con acrosport, que consiste en aprender y practicar diferentes figuras y pirámides básicas, en progresión de dificultad y de manera autónoma. El profesorado explicó los roles de portor, ágil y ayudante, recalcando la importancia del ayudante, y del montaje y desmontaje de las figuras de manera responsable. El dilema que inició el debate para el trabajo de la responsabilidad fue el siguiente: "Un entrenador es conocedor de la lesión de su jugador y de las ganas que tiene en participar en un partido de baloncesto (deporte elegido anteriormente). Es su jugador estrella y fundamental para ganar el partido, pero sabe que si se lo permite puede acabar lesionado de por vida. ¿Debería el entrenador permitirle continuar?”.

\subsection{Instrumentos}

El instrumento utilizado para realizar una evaluación pretest-postest del programa fue la versión española del Sport Value Questionnaire (SVQ-E; Cruz et al., 1999; Torregrosa y Lee, 2000). El SVQ-E se adaptó para este estudio añadiendo los valores Responsabilidad, Igualdad y Respeto, no contemplados en el cuestionario. Además, para facilitar la respuesta de los estudiantes con discapacidad intelectual se varió la escala de respuesta de siete puntos (siendo el uno lo contrario de lo que pienso, y hasta cinco, totalmente de acuerdo) por cinco (siendo uno nada importante para mí, y hasta cinco, muy importante), al igual que en el estudio de Torregrosa y Cruz (2009). Como resultado final se ha obtenido un instrumento que recoge las puntuaciones en referencia a 19 valores con un alpha de Cronbach de .845 , similar al referido por Torregrosa y Cruz (2009).

Además, con el objetivo de realizar una evaluación continua con carácter formativo, se utilizó, como instrumento de recogida de datos cualitativos, un registro de observación, donde se anotaron las respuestas y opiniones de los alumnos en los debates generados tras los dilemas.

(C) Ediciones Universidad de Salamanca / CC BY-NC-ND

Siglo Cero, vol. 48 (3), n. ${ }^{\circ}$ 263, 2017, julio-septiembre, pp. 73-86 


\section{LA PROMOCIÓN DE VALORES A TRAVÉS DE UN PROGRAMA DE ACTIVIDAD FÍSICA Y PREDEPORTES EN PERSONAS CON DISCAPACIDAD INTELECTUAL LEVE}

ELISA ISABEL SÁNCHEZ-ROMERO, M. ${ }^{a}$ PILAR VÍLCHEZ CONESA Y CRISTINA DE FRANCISCO PALACIOS

\section{Resultados}

Se llevaron a cabo análisis descriptivos con el objetivo de obtener los porcentajes de respuesta a los diferentes valores antes y después del programa, así como contrastes no paramétricos ( $t$ de Wilconxon) para analizar la eficacia del programa en relación a las medidas pre y post. También se realizaron pruebas de contraste paramétricas $(t$ de Student) para la comparación entre los valores incluidos en el programa de predeportes con los que no.

\subsection{Análisis de los valores antes y después del programa}

Respecto a los valores incluidos en el programa solo el valor honestidad/fair play obtuvo mejoras estadísticamente significativas $(Z=-2.07 ; p=.38)$. Este valor, en el pretest, fue considerado como nada importante por un $8.3 \%$ de los estudiantes, un $16.7 \%$ poco importante, un 50\% importante, un $8.3 \%$ bastante importante y un $16.7 \%$ muy importante. En el postest, ningún estudiante lo consideró nada importante, mientras que solo un $8.3 \%$ lo consideró poco importante, un $41.7 \%$ importante, un $16.7 \%$ bastante importante y un $33.3 \%$ muy importante.

El resto de valores, aunque no obtienen diferencias estadísticamente significativas entre el pre y el post, sí que presentan un incremento tras la aplicación del programa. En la Tabla 1 se exponen los resultados para cada valor antes (pre) y después del programa (post).

\begin{tabular}{|c|c|c|c|c|c|c|c|c|c|c|c|c|}
\hline & \multicolumn{2}{|c|}{$\begin{array}{c}\text { Compromiso } \\
(\%)\end{array}$} & \multicolumn{2}{|c|}{$\begin{array}{c}\text { COMPAÑERISMO } \\
(\%)\end{array}$} & \multicolumn{2}{|c|}{$\begin{array}{c}\text { RESPETO } \\
(\%)\end{array}$} & \multicolumn{2}{|c|}{$\begin{array}{c}\text { IGUALDAD } \\
(\%)\end{array}$} & \multicolumn{2}{|c|}{$\begin{array}{l}\text { HONESTIDAD/ } \\
\text { fair play }(\%)\end{array}$} & \multicolumn{2}{|c|}{$\begin{array}{c}\text { RESPONSABILIDAD } \\
(\%)\end{array}$} \\
\hline & Pre & Post & Pre & Post & Pre & Post & Pre & Post & Pre & Post & Pre & Post \\
\hline $\begin{array}{l}\text { Nada } \\
\text { importante }\end{array}$ & - & - & - & - & - & - & - & 8.3 & $8.3^{*}$ & - & - & - \\
\hline $\begin{array}{l}\text { Poco } \\
\text { importante }\end{array}$ & - & 8.3 & 8.3 & - & - & - & 8.3 & - & $16.7^{*}$ & $8.3^{*}$ & - & - \\
\hline Importante & 8.3 & 16.7 & 16.7 & 16.7 & 16.7 & 8.3 & 16.7 & 8.3 & $50 \%$ & $41.7^{*}$ & 8.3 & 16.7 \\
\hline $\begin{array}{l}\text { Bastante } \\
\text { importante }\end{array}$ & 16.7 & 8.3 & 16.7 & 16.7 & 33.3 & 16.7 & 25 & 25 & $8.3^{*}$ & $16.7^{* *}$ & - & - \\
\hline $\begin{array}{l}\text { Muy } \\
\text { importante }\end{array}$ & 75 & 66.7 & 58.3 & 66.7 & 50 & 75 & 50 & 58.3 & $16.7 \%$ & $33.3^{*}$ & 91.7 & 83.3 \\
\hline
\end{tabular}

$* \mathrm{p}<.05$.

En la Tabla 1 se exponen los resultados para cada valor antes (pre) y después del programa (post), mostrando la puntuación de la percepción personal de los valores trabajados. 
LA PROMOCIÓN DE VALORES A TRAVÉS DE UN PROGRAMA DE ACTIVIDAD FÍSICA Y PREDEPORTES EN PERSONAS CON DISCAPACIDAD INTELECTUAL LEVE

ELISA ISABEL SÁNCHEZ-ROMERO, M. ${ }^{a}$ PILAR VÍLCHEZ CONESA Y CRISTINA DE FRANCISCO PALACIOS

\subsection{Comparación de los valores incluidos y los no incluidos en el programa}

En este apartado se muestran los resultados entre los valores que se trabajaron en el programa de predeportes (compromiso, compañerismo, respeto, igualdad, honestidad/fair play y responsabilidad) y los valores que no se incluyeron en el programa (aceptación, autorrealización, ayuda/compasión, cohesión, conformidad, deportividad, diversión, emoción/excitación, gustar/imagen pública, justicia, logro personal, mantenimiento de contrato, obediencia, salud y forma física).

Los valores trabajados obtuvieron una media en la valoración de los participantes de $4.36 \pm 0.34$, mientras que en los no trabajados se encontró una media de $3.52 \pm 0.59$. La prueba $t$ para la comparación de las medias resultó estadísticamente significativa $(t=3.15 ; p<.01)$. Estos datos fueron obtenidos tras la comprobación de los supuestos de homogeneidad de varianzas $(F=1.39 ; p=.254)$ y de normalidad $(Z=.480 ; \mathrm{p}=.975)$.

\subsection{Descripción de la observación de los alumnos tras los dilemas presentados}

Compromiso. Se observa la congruencia entre las respuestas de los alumnos al dilema teórico y la situación deportiva vivida por ellos. Se les pregunta qué valor creen que están trabajando y cómo se traslada al aula y al futuro mundo laboral. Dudan entre compromiso y compañerismo, por lo que el profesorado les explica que el compromiso implica que cada uno debe ser consciente de la importancia que tiene el cumplir con las tareas asignadas o adquiridas con una persona por encima de sus propios intereses. Respecto al compañerismo les dice que se caracteriza por la comprensión, el apoyo y la ayuda, sin buscar nada a cambio, con las personas con las que se convive. Entonces, para asegurarse de que sí lo han entendido, pregunta a la clase sobre cómo se lleva a cabo el compromiso en su aula real. Acto seguido, los alumnos refieren que deben mostrarse comprometidos con los profesores haciendo los deberes, llegando puntuales al aula, etc., al igual que en un futuro trabajo.

Compañerismo. Tras enunciar este dilema, los alumnos dudan de cómo actuarían. Algunos comentan que por ser compañeros deberían ayudar a la compañera y otros argumentan que entonces estarían desobedeciendo a la profesora, por lo que estarían faltando al respeto a esta. Para ayudarles a descubrir el valor que se está trabajando, se les recuerda que, jugando al bádminton, había cuatro compañeros que tenían dificultades para darle al volante, por lo que empezaron trabajando por separado para mejorar su técnica y después se integraron en los grupos con los demás compañeros para hacer la prueba de evaluación. Es importante destacar que todos habían mostrado compañerismo a pesar de las intervenciones de los árbitros sugeridas por los docentes, recordándoles que si no jugaban no podrían puntuar los partidos y ver quién ganaba. Los alumnos comentan que debían dejar jugar a los compañeros con menos habilidad, porque, si no, serían malos compañeros. Se les pregunta entonces qué valor se puede ver en sus comportamientos, a lo que contestan que el compañerismo. Cuando se les confirma que efectivamente ese era el valor (aunque el respeto se refleja también en el dilema), se les pregunta por situaciones en el aula donde también se observa el 
compañerismo. Responden con comentarios tales como no interrumpir al compañero cuando habla en clase para dar la respuesta correcta antes que él, ayudar cuando no sabe hacer alguna tarea, no burlarse cuando hay una intervención incorrecta, etc. Ante la pregunta de cómo podrían ser buenos compañeros en el trabajo, dicen que igual, pero añaden que haciendo sus tareas a tiempo para no perjudicar las obligaciones de los otros compañeros.

Respeto. Tras la presentación del dilema de dicho valor, todos dicen que la protagonista no debe seguir insistiendo porque el árbitro puede expulsarla. Se les intenta hacer pensar sobre si no hay una razón por no insistir que se relacione con los valores. Quedan un poco desconcertados porque no ven ningún valor. Se les orienta hacia la figura del árbitro, que es como un profesor en el aula y como un jefe en el trabajo, “¿qué tienen en común? Son figuras de autoridad a las que hay que...” (los alumnos responden tras una pausa) respetar. En la situación de juego, se dividieron en dos equipos de cinco con un árbitro que debía alternarse. Para forzar situaciones conflictivas con el árbitro se le dieron indicaciones de que fuese muy duro y de que expulsase a los jugadores al mínimo comentario a sus decisiones. Esto provocó que seis alumnos se enfrentasen entre sí cuando ejercieron de árbitro. A estos se les pidió que contasen cómo se habían sentido ante los reproches de sus compañeros para que todos supiesen qué es lo que se siente cuando se le falta el respeto a una persona.

Igualdad. Únicamente dos alumnos se posicionan en que el entrenador no debe sacar a la protagonista, que si ésta no sabe jugar no debe salir porque perjudica al equipo. Se les recuerda la situación de competición en la que uno de los alumnos evitaba pasar el balón a los compañeros con menos habilidad, sobre todo a las chicas. Cuando finalmente los estudiantes logran identificar el valor de la igualdad, estos dos alumnos se reafirman en que no hay igualdad posible. Uno de los docentes les pregunta si eso es así en la vida diaria o solo en el deporte. Uno de los alumnos señalados anteriormente dice que las mujeres tienen que dedicarse a sus cosas, esto es, hacer la comida, planchar, etc., tras lo cual el otro alumno asiente. Ante esto, algunos alumnos debaten con sus compañeros el tema de la igualdad sin llegar a un acuerdo.

Honestidad/fair play. Los estudiantes comentan que quizá el protagonista no disfrutaba de su victoria porque había ganado con trampas. Uno de los profesores les aclara que jugar limpio o con deportividad es jugar sin hacer ningún tipo de trampa, sin intentar engañar al árbitro, respetando al compañero, tratando al contrario sin groserías, etc. Que a esto también se le llama fair play y que incluye varios de los valores hasta ahora tratados. En relación a la situación deportiva con el "fut-tenis" se les instó a recordar lo ocurrido respecto a la validez de las puntuaciones (el jugador que tenía más cerca la línea tenía que decir si había sido dentro o fuera). Durante este juego no hubo conflictos porque todos aceptaban la decisión del jugador, incluso si este no lo había visto, todos llegaban a un acuerdo, poniendo en práctica el juego limpio.

Responsabilidad. Tras el dilema del valor responsabilidad, tres alumnos responden que si fuesen el jugador competirían a pesar de la lesión, pero que si fueran entrenadores no obligarían al jugador a competir. El resto de los alumnos no se arriesgarían a competir (siendo deportista) ni obligarían al deportista a competir (siendo entrenador). Respecto a la situación deportiva dentro del acrosport, el profesorado 
LA PROMOCIÓN DE VALORES A TRAVÉS DE UN PROGRAMA DE ACTIVIDAD FÍSICA Y PREDEPORTES

EN PERSONAS CON DISCAPACIDAD INTELECTUAL LEVE

ELISA ISABEL SÁNCHEZ-ROMERO, M. ${ }^{\mathrm{a}}$ PILAR VÍLCHEZ CONESA Y CRISTINA DE FRANCISCO PALACIOS

les recuerda la responsabilidad de cada uno de los miembros, puesto que, si alguno fallaba, no era posible llevar a cabo la figura o alguno se podía hacer daño, pues la responsabilidad supone asumir las consecuencias de las propias decisiones y actos.

\section{Discusión}

Los objetivos del presente trabajo eran dos: 1) promover los valores a través de un programa de actividad física y predeportes en personas con discapacidad intelectual leve, y 2) comparar el trabajo de estos valores a través del programa de actividad física y predeportes con otros valores no incluidos. Tras los resultados obtenidos, los alumnos mostraron mayor consideración por los valores trabajados tras el programa de actividad física y predeportes, aunque no presentase significatividad estadística, quizá debido al reducido tamaño muestral. En este sentido coincidimos con Borrás et al. (2009), puesto que la idea tradicional de que los valores son principios inmutables podría ponerse en duda, debido a los resultados indicados en la Tabla 1, donde se muestra un leve incremento en la percepción personal de los alumnos respecto a la importancia de los valores trabajados.

Respecto al valor compromiso, se encuentra un leve incremento respecto a la percepción de importancia del alumnado. En esta línea Borrás et al. (2009) realizaron una investigación con jugadores cadetes de primera regional, mostrando el valor mantenimiento de contrato (entendido por dichos autores de forma similar a como se entendió el valor compromiso en la presente investigación) como uno de los valores que pasó de ser puntuado bastante importante a muy importante.

Se corrobora con otros estudios que valores como el compañerismo y el respeto se trabajan de manera importante desde la práctica de actividad física educativa (Martínez et al., 2014). Según Borrás et al. (2009), el valor compañerismo es uno de los valores que más mejora su puntuación en adolescentes tras un programa de deportes. Por otro lado, el respeto es uno de los valores más percibidos por los alumnos en Educación Física (Sánchez-Oliva, Leo, Amado, González-Ponce y López, 2012). En la educación superior, también hay estudios que perciben el respeto hacia sus compañeros como uno de los valores adquiridos durante la práctica de educación física (Ionescu, 2012).

En el valor igualdad, se incrementó la percepción de importancia de los alumnos, aunque no de forma significativa, ya que en un primer momento el $50 \%$ de los alumnos lo puntuaron como importante, pasando a puntuarlo un $58.3 \%$ muy importante tras la implementación del programa. Estos resultados alientan a seguir trabajando dicho valor, ya que, en el contexto educativo, la igualdad es un valor percibido como prioritario por los docentes de primaria (Rebollo, García-Pérez, Piedra y Vega, 2011).

En el valor honestidad/fair play, las diferencias fueron estadísticamente significativas, sin considerar tras la intervención ningún estudiante que este valor fuese nada importante, y aumentando aquellos que lo consideraron bastante importante y muy importante. Al igual que en el presente estudio, las opiniones y las conductas de niños de Primaria y de Secundaria mejoraron tras la aplicación de un programa de intervención 
para desarrollar la responsabilidad en los comportamientos de fair play (Cecchini, Montero y Peña, 2003; Cecchini, Fernández y González, 2008). Asimismo, los expertos en Educación Física también consideran este valor como importante, aunque por detrás de otros como la responsabilidad y el respeto (Martínez et al., 2014).

Por otro lado, la responsabilidad es un valor importante para trabajar en Educación Física y en programas como el que aquí se presenta, coincidiendo con Nicoleta (2014), cuando afirma que la Educación Física y el deporte contribuyen a la educación óptima y la formación social de la personalidad, adquiriendo responsabilidad y participación activa en la resolución de problemas en la sociedad. También hay valores que los alumnos no perciben a priori como muy importantes en la actividad física educativa, pero sí lo hacen los expertos y profesores de Educación Física, según afirman Martínez et al. (2014), como son los valores de responsabilidad y respeto. En esta misma línea se sitúan Pazo y Tejada (2015), al entrevistar a técnicos deportivos de alto rendimiento, afirman que estos situaron el valor de responsabilidad entre los primeros puestos en cuanto a importancia de una lista de valores dada, aunque no todos lo consideraban imprescindible. En el contexto educativo, los padres y madres de alumnos de $3 .^{\circ} \mathrm{y}$ de $4 .^{\circ}$ de Educación Secundaria coincidieron en que la responsabilidad es un valor prioritario en la educación formal de sus hijos, con un $67 \%$ y $93 \%$, respectivamente (López-Lorca, 2004).

Así mismo, se obtuvieron mejoras significativas en los valores trabajados a través del programa en comparación con los valores no incluidos en el programa, corroborando así lo que también obtuvieron otros estudios que trabajaron valores a través de la práctica físico-deportiva (Borrás et al., 2009).

El campo de los valores no ha sido objeto de investigación suficiente (Torregrosa y Lee, 2000), a pesar de los beneficios que puede aportar el desarrollo e implementación de programas educativos para la adquisición de valores, a través de la práctica deportiva. Prueba de ello fue el Programa Delfos, para el desarrollo de valores en alumnos de secundaria a través del deporte (Cecchini et al., 2008). Tras llevarse a cabo durante un curso escolar, se observó la adquisición de valores en el alumnado, traduciéndose en disminución de los comportamientos violentos dentro del aula, así como la adquisición de valores que los alumnos transfirieron a otros contextos no deportivos. Šukys y Majauskienè (2014) midieron los efectos de un programa de educación olímpica en alumnos de edades comprendidas entre los 13 y los 18 años, concluyendo que la implementación de dicho programa anotó un desarrollo mayor de valores tales como la solidaridad o la convivencia, entre otros. Leonte (2014), por su parte, observó la relación entre realizar actividades deportivas y la adquisición de valores en adolescentes mujeres de entre 16 y 18 años, las cuales indicaron que la práctica deportiva producía mejoras en la realización personal, así como en valores tales como el respeto y la integración.

Una enseñanza sin tener en cuenta la dimensión moral no será una enseñanza completa (Binaburo y Muñoz, 2007). La educación en valores no puede asemejarse a la enseñanza tradicional de transmisión de conocimiento, sino que exige la experiencia del valor (Ortega y Mínguez, 2001). A través de estructuras cooperativas en el deporte escolar pueden fomentarse la educación en valores en el alumnado (González, Cecchini, Fernández-Río y Méndez, 2008). Aquí reside la importancia de programas 
educativos como el que se presenta, al asociar el trabajo de valores morales con la experiencia práctica de los mismos que el deporte y la actividad física conllevan, coincidiendo con Monjas, Ponce y Gea (2015), al indicar la idoneidad de los programas deportivos para promover valores en el ámbito escolar.

\section{Conclusiones}

Los valores trabajados en el programa de actividad física y predeportes muestran valores más altos de importancia por parte de los participantes, aunque solo el valor honestidad/fair play obtuvo significatividad estadística. Por tanto, las opiniones de los alumnos recogen la pertinencia del programa implantado, aunque solo de forma clara en honestidad.

Los valores incluidos en el programa obtuvieron puntuaciones más elevadas respecto a la importancia dada por los participantes en comparación a los valores que no se trabajaron en el programa, con diferencias estadísticamente significativas, poniendo de manifiesto la posible influencia del programa en la percepción de los estudiantes.

Las reflexiones realizadas por los alumnos en los momentos de debate, tras la lectura de los dilemas morales, muestran que estos fueron capaces de entender los valores y lo aprendido en las sesiones de predeporte en otras situaciones de su vida diaria.

\section{Referencias bibliográficas}

Barba, B. y Matías, J. (2005). Desarrollo del juicio moral en la educación superior. Revista Mexicana de Investigación Educativa, 10 (24), 67-92.

Barba, F. J., Barba, F. J. y Muriarte, D. (2003). Desarrollo moral en Educación Física. Una propuesta de dilemas morales a través de la actividad física y el deporte. Revista Digital efdeportes, 61. Recuperado de http://www.efdeportes.com/efd61/moral.htm.

Benítez, A. (2009). La Educación en valores en el ámbito de la Educación Superior. Revista Iberoamericana de Calidad, Eficacia y Cambio en Educación, 7 (2), 116-129.

Binaburo, J. A. y Muñoz, B. (2007). Educar desde el conflicto. Guía para la mediación escolar. Barcelona: CEAC.

Borrás, P. A., Palou, P., Ponseti, F. X., Vidal, J. y García-Mas, A. (2009). La educación en valores en la práctica deportiva de los adolescentes: efectos de una intervención para la promoción de la deportividad sobre la estructura de valores de los deportistas. Revista Española de Pedagogía, 243, 355-370.

Cecchini, J. A., Fernández, J. y GonzÁlez, C. (2008). Repercusiones del Programa Delfos de educación en valores a través del deporte en jóvenes escolares. Revista de Educación, 346, 167-186.

Cecchini, J. A., Montero, J. y Peña, J. V. (2003). Repercusiones del programa de intervención para desarrollar la responsabilidad personal y social de Hellison sobre los comportamientos de fair-play y el auto-control. Psicothema, 15 (4), 631-637.

Cerrillo, R., IzuzQuiza, D. y Egido, I. (2013). Inclusión de jóvenes con discapacidad intelectual en la Universidad. Revista de Investigación en Educación, 11 (1), 41-57. 
LA PROMOCIÓN DE VALORES A TRAVÉS DE UN PROGRAMA DE ACTIVIDAD FÍSICA Y PREDEPORTES EN PERSONAS CON DISCAPACIDAD INTELECTUAL LEVE

ELISA ISABEL SÁNCHEZ-ROMERO, M. $^{a}$ PILAR VÍLCHEZ CONESA Y CRISTINA DE FRANCISCO PALACIOS

Cruz, J., Boixadós, M., Capdevila, L., Mimbrero, J., Torregrosa, M. y Valiente, L. (1999). Evaluación del fairplay en el deporte profesional y de iniciación. En CONSEJO SUPERIOR DE DePORTES (Ed.), Participación deportiva:perspectiva ambiental y organizacional (pp. 11-52). Madrid: Consejo Superior de Deportes.

Cruz, J., Boixadós, M., Valiente, L. y Torregrosa, M. (2001). ¿Se pierde el “fairplay” y la deportividad en el deporte en edad escolar? Apunts. Educación Física y Deportes, 2 (64), 6-16.

Firestone, W. A. y Pennell, J. R. (1993). Teacher commitment, working conditions, and differential incentive policies. Review of Educational Research, 63 (4), 489-525.

Fullana, J., Pallisera, M., Martín, R., Ferrer, C. y Puyaltó, C. (2015). La transición a la vida adulta de jóvenes con discapacidad intelectual. Evaluación de un programa de formación para la mejora de las competencias personales. Revista de Investigación en Educación, 13 (1), 53-68.

García-Hoz, V. (1993). Tratado de Educación Personalizada. Introducción general a una pedagogía de la persona. Madrid: RIALP.

González, C., Cecchini, J. A., Fernández-Río, J. y Méndez, A. (2008). Posibilidades del modelo comprensivo y del aprendizaje cooperativo para la enseñanza deportiva en el contexto educativo. Aula Abierta, 36 (1-2), 27-38.

Hall, B. P. (1995). Values Shift. A Guide to Personal \& Organizational Transformation. Rockport, MA: Twin Lights.

Halstead, J. M. y TAYlor, M. J. (2000). The development of values, attitudes and personal qualities. A review of recent research. Slough: NFER.

Hernández-Mendo, A. y Planchuelo, L. (2014). El incremento del desarrollo moral en las clases de educación física. Revista Iberoamericana de Psicología del Ejercicio y el Deporte, 9 (2), 369-392.

IONESCU, C. L. (2012). Social values reflection effect of students conduct practice motor activities. Sport Society: Internacional Journal of Physical Education and Sport, 13 (1), 11-31.

IZuZQuizA, D. (2012). El valor de la inclusión educativa de jóvenes con discapacidad intelectual en las instituciones de educación superior: El Programa Promentor. Bordón, 64 (1), 109-125.

Langdon, P. E., Clare, I. C. H. y Murphy, G. H. (2010). Developing an understanding of the literature relating to the moral development of people with intellectual disabilities. Developmental Review, 60 (3), 273-293.

LEONTE, N. (2014). The relationship between sport activities-effects-values in secondary school education. Gymnasium: Scientific Journal of Education, Sports and Health, 15 (1), 72-81.

López-Lorca, H. (2004). Padres y alumnos ante el valor de responsabilidad. Educatio, 22, 187-205.

Martínez, R., Cepero, M., Collado, D., Padial, R., Pérez, A. y Palomares, J. (2014). Acquisition of values and attitudes across games and sports in physical education, in the Secondary Education. Journal of Sport and Health Research, 6 (3), 207-216.

Molina, V., Vargas, A. y SAn Martín, V. (2014). Bioética: Discapacidad y actividad física. Revista de Ciencias de la Actividad Física UCM, 15 (2), 73-85.

Naval, C. (2005). Ámbito familiar: confianza y respeto. En A. Bernal (Ed.), La Familia como ámbito Educativo (pp. 145-162). Madrid: RIALP.

NicoletA, L. (2014). The relationship between sport activities-effects-values in secondary school education. Gymnasium: Scientific Journal of Education, Sports, and Health, 1 (XV), 72-81.

Ortega, P. y Mínguez, R. (2001). Los valores en la educación. Barcelona: Ariel Educación.

(C) Ediciones Universidad de Salamanca / CC BY-NC-ND

Siglo Cero, vol. 48 (3), n. ${ }^{\circ}$ 263, 2017, julio-septiembre, pp. 73-86 
Ortega, P. y Mínguez, R. (2003). Familia y transmisión de valores. Revista Interuniversitaria de Teoría de la Educación, 15, 33-56.

Otero-Pazos, M., Navarro-Patón, R. y Abelairas-Gómez, C. (2014). El deporte escolar en función del género desde el punto de vista del profesorado. Trances, 6 (5), 289-310.

Parisi, I., Mouratidou, K., Koidou, E., Tsorbatzoudis, H. y Karamavrou, S. (2015). Effects of motivational climate, type of school and gender on students' moral competences in their daily life and physical education. Trends in Sport Sciences, 1 (22), 39-46.

Pazo, C. I. y TejadA, J. (2015). Los valores individuales y sociales según los profesionales de la educación física y del fútbol onubense cadete. e-balonmano.com. Revista de Ciencias del Deporte, 11 (1), 83-98.

Peter, I. (2014). Reconsidering Place of Traditional Institutions under the Nigerian Constitution: A Comparative Analysis. Journal of Law, Policy and Globalization, 31, 135-148.

Pombo, I. y Fantova, F. (2011). La Administración Pública ante los desafíos de la Ética. Siglo Cero, 42 (237), 16-20.

Rebollo, M. A., García-Pérez, R., Piedra, J. y Vega, L. (2011). Diagnóstico de la cultura de género en educación: actitudes del profesorado hacia la igualdad. Revista de Educación, 355, 521-546.

SÁnchez-Oliva, D., Leo, F. M., Amado, D., González-Ponce, I. y López, J. M. (2012). Differential Analysis of perception of the development of values in Physical Education clases. Retos, 22, 9-12.

Schalock, R. L. y Verdugo, M. Á. (2007). El concepto de calidad de vida en los servicios y apoyos para personas con discapacidad intelectual. Siglo Cero, 38 (224), 21-36.

ŠUKYs, S. y MAJAuSKIENE, D. (2014). Effects of an integrated olympic education program on adolescent athletes' values and sport behavior. Social Behavior and Personality, 42 (5), 811822. http://dx.doi.org/10.2224/sbp.2014.42.5.811.

Torregrosa, M., y CruZ, J. (2009). Entusiastas, aficionados y espectadores: sus valores, motivaciones y compromiso. CCD: Cultura, Ciencia y Deporte, 4 (12), 149-157.

Torregrosa, M. y Lee, M. (2000). El estudio de los valores en psicología del deporte. Revista de Psicología del Deporte, 9 (1-2), 71-83. 